\title{
Factores que inciden en la acción de escribir a mano
}

Sylvia Cristina Duarte Torres, T.O.* e-mail: ocupacional@tutopia.com

R. MINS. 6870

\section{RESUMEN}

El presente articulo constituye un documento que invita al Terapeuta Ocupacional al análisis de las conductas sensoriomotoras que hacen referencia a la actividad realizada con miembros superiores, propia del ser humano, $y$ que hace parte de su vida cotidiana, escribir a mano; se hace una revisión bibliográfica del desarrollo de la praxis manual, tipos de agarres, disfunciones y factores que se recomiendan tener en cuenta para medir la efectividad de la calidad de la prehensión de la mano, los diferentes movimientos que intervienen para lograr la coordinación, la destreza y la manipulación del objeto.

Palabras claves: Ocupación. Función de la mano, escribir con lápiz.

Los diferentes factores que están involucrados en la habilidad de los movimientos de la mano, le permiten al ser humano, interactuar con los diferentes objetos, logrando una destreza de tan alto nivel que con el transcurso del tiempo la persona no percibe la exactitud de la misma y el control del sistema nervioso central.

La función de la extremidad superior, esta dada por la sensación, movilidad y estabilidad del segmento en su totalidad, pero ante todo por la voluntad del individuo, su motivación y deseo de desarrollar habilidades con precisión, velocidad y fuerza muscular se suman al refinamiento y maduración de las zonas cerebrales y las vías cortico-espinales.

\footnotetext{
* Terapeuta Ocupacional, egresada de la Escuela Colombiana de Rehabilitación afiliada al Colegio Mayor del Rosario, Docente Escuela Colombiana de Rehabilitación.
} 


\section{REVISIÓN DE LA LITERATURA}

\section{Desarrollo embriológico}

Existe un ciclo de tiempo donde se manifiesta el comienzo del desarrollo de la conducta práxico manual, hecho que permite establecer etapas dentro de la vida embriológica.

Es importante tener en cuenta todas las estructuras anatómicas y el mecanismo kinesiológico de la mano, dado que por esto desde el desarrollo embriológico se debe comprender la magnitud de la función de la mano y el brazo.

En primera instancia en la formación estructural se establece que a las 4 semanas de gestación aparece como cartilago la escápula, el húmero, el radio y el cubito; a la 6 semana aparece el tercer digito, los metacarpianos.

La 7 semana comienza a ser muy importante en el ámbito de movimiento debido a que el brazo y antebrazo realizan la rotación interna y externa, a la 8 semana de gestación se observa la separación lateral del pulgar u oposición móvil del mismo, lográndose la adquisición del primer organizador de la praxis manual.

En la 12 semana se logra la flexión de las manos, como segundo organizador, y el tercero es a la 16 semana donde el niño logra abrir y cerrar las manos.

Con una rapidez asombrosa las estructuras toman forma permitiendo la integración de la mano al sistema neuro-motor adquiriendo movimientos suaves, refinadosy de perfeccionamiento.

\section{PRAXIS MANUAL}

La definición de praxis manual esta enfocada a la habilidad de realizar un movimiento pequeño, preciso y delicado, demostrando el grado de perfeccionamiento alcanzado en el dominio de la actividad voluntaria.

Durante las primeras etapas de la vida, la actividad es global, difusa, no existe la maduración neuromuscular para lograr un acto voluntario independiente; la evolu- 
ción de la praxis manual permite identificar 4 patrones a ser considerados: alcanzar, agarrar, sostener y manipular.

\section{Alcanzar:}

Incluye movimientos de brazo, antebrazo y mano, es una acción que pone la mano en contacto con el ambiente, para que las respuestas de los agarres se puedan desarrollar y adaptar a funciones especificas..

El alcance requiere de la separación del miembro superior del resto del cuerpo, así como del control proximal de la escápula para acomodarse a las dimensiones espaciales y temporales del movimiento. En supino el alcance empieza con el golpeteo de objetos, primero al lado y luego en la mitad, contribuyendo al desarrollo de la flexo-extensión del miembro superior en contra de la gravedad y la conducta de llevar las manos a línea media.

En prono el alcance adquiere una importancia en la conducta de mantener y sostener peso de un lado mientras el otro esta libre para alcanzar juguetes en plano frontal favoreciendo la extensión en contra de la gravedad. A medida que el niño adapta el alcance para moverse y recurrir al juguete los patrones de alcance se diferencian en el arrastre y en el gateo; En conclusión, al estar diferenciados los alcances desde la posición supina, prona o secuencia de rolado, los patrones de equilibrio y de estabilidad en línea media hace que el individuo realice un alcance con propósito en todos los planos de movimiento.

\section{Soltar:}

El soltar se define como la habilidad de liberar un objeto que se sostiene en la palma de la mano, se da por la combinación del patrón extensor.

Después de los 4 meses el niño realiza un soltar involuntario como resultado del movimiento general de su miembro superior o estimulo táctil; a los 6 ó 7 meses el niño inicia transferir objetos de una mano a la otra, siendo inicialmente halado de la mano que lo sostiene, observándose un soltar y un halar que se desarrollan simultáneamente.

A los 8 meses representa el comienzo del soltar voluntario que sumado a la relación causa-efecto y permanencia del objeto, se especializa la extensión voluntaria de los dedos. 


\section{Agarre y manipulación:}

La función prensil de la mano depende de la integridad cinética, de la estabilidad proximal de antebrazo y del balance de los músculos sinergístas y los antagonistas entre los extrínsecos y los intrínsecos.

Los diferentes patrones de la mano han sido descritos por Napier (1956) y por Flatt (1972) los cuales han considerado:

- Los agarres de fuerza: combinan la fuerza del pulgar en flexión, aducción y la fuerza de los otros cuatro dígitos hacia el lado cubital, con extensión de muñeca y estabilización del objeto en la palma.

- Los agarres de precisión: la muñeca deja de ser tan importante el pulgar trabaja en oposición y los demás dígitos en semiflexión.

La mayoría de actividades instrumentales de la vida diaria, requieren de combinaciones de agarres fuertes y de precisión, tales como abrir un frasco, martillar una puntilla entre otros, mientras que la actividad de escribir a mano específicamente accionar un lápiz es una actividad de precisión pero que necesita de la estabilidad especifica de los patrones de fuerza.

Los factores sensorio -integrativos están referidos al sistema táctil que le permite desarrollar la habilidad de la esterognosia, la información háptica le permite explorar desde el primer año de vida la forma y las características de los juguetes y aprender del medio ambiente, de su cuerpo y del efecto que tienen sus movimientos. La integración entre la percepción visual y háptica esta dada a partir del 6 mes pues el reconocer la forma del objeto visualmente facilita el contacto táctil de éste. Piaget y Inhelder (1984) de acuerdo a las investigaciones realizadas con niños entre 2 y 7 años, determinaron que esta habilidad háptica es progresiva y se incrementa con la edad; por ejemplo entre los 30 meses y los 42 meses el niño es capaz de reconocer correctamente objetos comunes pero no identifica formas, entre los 42 meses y los 60 meses el niño identifica correctamente formas topológicas y figuras geométricas.

La discriminación háptica de textura, tamaño y peso se manifiesta a partir de los 4 años de edad y su mayor desarrollo va hasta los 9 años. 
El sistema propioceptivo da información sobre el sentido de la posición de la mano y del objeto con respecto a la mano, participando en la preparación de la mano para el agarre y en la manipulación del objeto, permitiendo regular la fuerza de prehensión durante el agarre, ajustando las estructuras a la situación correcta de los programas motores, siendo controlado por el cerebelo, interviniendo en la planeación del movimiento y permitiendo asegurar la calidad, exactitud y precisión del mismo.

La habilidad de la cinestesia y sistema visual contribuyen con todas las funciones de índole perceptual logrando de esta manera un proceso que interrelaciona la esterognosia con la demás información sensorial lleva al manejo de la herramienta o el objeto que se tiene en la mano, modulando la fuerza necesaria para manejarlo y la presión que se debe ejercer sobre él según las características del mismo, la cinestesia permite la planeación motora del acto, la organización y asociación del mismo y por ende la acción en si (el propósito); la percepción visual contribuye con la organización espacial del instrumento.

Realizando un examen cuidadoso de los diferentes autores que han investigado sobre la praxis manual se encuentran que en el ámbito del neurodesarrollo es importante establecer que:

1. La información somatosensorial se codifica a lo largo de la corteza posterior, desde la zona primaria sensorial hasta la parte posterior del lóbulo parietal (Jones y Powell, 1970), permitiendo integrar combinaciones, visuales, táctiles y kinestésicas que le posibilitan desarrollar movimientos muy finos, armónicos y coordinados de la mano.

2. Las vías vestíbulo-espinales y retículo-espinales controlan los movimientos proximales de los segmentos, estas estructuras subcorticales al ser mielinizadas establecen la estabilidad escapular para desarrollar movimientos como el lanzar y el alcanzar; de esta manera permite el desarrollo de los movimientos distales de la mano.

3. El otro aspecto a tener en cuenta es la escala de SCNECK Y HENDERSON (1991 y 1990) que establecen los patrones de prehensión o agarre de tipo primarios o primitivos, transicionales y maduros. 


\section{Cuadro 1.}

\begin{tabular}{|c|c|c|}
\hline TIPO DE AGARRE & NIVEL & DESCRIPCIÓN \\
\hline 1. Agarre palmar radial & Primitivo & $\begin{array}{l}\text { Antebrazo pronado, movimiento de todo el brazo, el } \\
\text { pulgar hacia abajo. }\end{array}$ \\
\hline 2. Agarre palmar supinado & Primitivo & $\begin{array}{l}\text { Muñeca flexionada, movimiento del todo el brazo, } \\
\text { pulgar hacia arriba. }\end{array}$ \\
\hline 3. Agarre pronado digital & Primitivo & $\begin{array}{l}\text { El dedo índice esta extendido, el antebrazo no es } \\
\text { estable. }\end{array}$ \\
\hline 4. Agarre de cepillo & Primitivo & $\begin{array}{l}\text { Es realizado con los dedos, mano pronada, con mo- } \\
\text { vimientos de muneca, el objeto se posiciona en la } \\
\text { palma, el antebrazo en el aire y movimientos de } \\
\text { brazo. }\end{array}$ \\
\hline $\begin{array}{l}\text { 5. Agarre con dedos } \\
\text { extendidos }\end{array}$ & Primitivo & $\begin{array}{l}\text { La muñeca esta estable, y pronada, con ligera desvia- } \\
\text { ción cubital y mueve el antebrazo como un todo. }\end{array}$ \\
\hline 6. Agarre con pulgar & Transicional & $\begin{array}{l}\text { El objeto se ajusta contra el pulgar y la falange media } \\
\text { del dedo indice, el pulgar cruza el lápiz y los demás } \\
\text { dedos parecen escondidos, mueve muñeca y dedos, } \\
\text { el antebrazo se posiciona en la mesa. }\end{array}$ \\
\hline 7. Agarre trípode estática & Transicional & $\begin{array}{l}\text { Estabiliza el objeto en el lado radial con apoyo del II } \\
\text { y III dedo, el pulgar estabiliza la completa oposición, } \\
\text { muñeca ligeramente extendida, se mueve la mano } \\
\text { como una unidad. El antebrazo descansa en la mesa. }\end{array}$ \\
\hline 8. Agarre de 4 puntos & Transicional & $\begin{array}{l}\text { Los } 4 \text { primeros dedos en oposición, movimiento de } \\
\text { muñeca y dedos, antebrazo estable en mesa. }\end{array}$ \\
\hline 9. Agarre trípode lateral & Maduro & $\begin{array}{l}\text { Estabiliza el objeto entre el lll dedo y el pulpejo del } \\
\text { pulgar, el pulpejo del índice es parte del equipo y } 4 \text { y } 5 \\
\text { dedo estabilizan la articulación metacarpofalangica }\end{array}$ \\
\hline 10. Agarre trípode dinámico & Maduro & $\begin{array}{l}\text { El objeto se estabiliza oposicionando el pulgar entre } \\
\text { el pulpejo del II dedo y apoyo en el III. }\end{array}$ \\
\hline
\end{tabular}

Esta escala le permite al Terapeuta establecer el nivel de Prehensión que posee la persona, la forma de interactuar con el objeto y establecer si el agarre esta siendo funcional, si contribuye a cumplir con los factores de precisión, velocidad, modulación de la fuerza, equilibrio entre músculos extrínsecos e intrínsecos y legibilidad de los signos realizados.

Dentro de los mecanismos neuromusculares se ha encontrado en la literatura que la función de la mano esta dada por un buen control postural, simetría y alineación 
del cuerpo con respecto a la cabeza, por lo tanto la presencia de un reflejo tónico nucal asimétrico incide directamente en la ejecución de las actividades bilaterales. Un adecuado tono muscular permite que la persona pueda realizar ajustes posturales oportunos para la acomodación de los materiales y herramientas de la actividad; el desarrollo de los músculos extrínsecos le permiten al hombre desarrollar la fuerza necesaria y el desarrollo de los músculos intrínsecos facilita la adquisición de la precisión de los agarres más finos o los utilizados para las actividades livianas.

\section{LA ACCIÓN DE ESCRIBIR A MANO}

La escritura es una habilidad muy importante en los niños de edad escolar, es la actividad central, producir una escritura legible, fluida para poder expresar, comunicar y registrar sus ideas y sus logros académicos.

El agarre del lápiz es uno de los aspectos de la escritura que debe ser muy cuidadosamente estudiado por los Terapeutas ocupacionales que trabajan con niños, a este factor se le une el ergonómico que hace referencia a la interacción y ajuste entre las habilidades del niño (madurez neurológica, motora y sensorial) y las características de la herramienta como es la forma del lápiz.

La intervención involucra el empleo de herramientas adaptadas, adaptadores para el lápiz, uso de flumaster en vez de lápiz, papel con renglones más anchos, etc.

Muchos niños entre los 4 y los 6 años de edad desarrollan un agarre típico de la herramienta que es la pinza trípode dinámica, en la medida que van creciendo parecen refinar este agarre; sin embargo en la mayoría de niños que presentan dificultades en la escritura se observa variaciones en el tipo agarre del lápiz, sin que esto se encuentre muy claro.

La manipulación eficiente del lápiz depende de la necesidad de saber diferenciar los movimientos individuales de los dedos, desempeñar la acción con velocidad y tener una fuerza de agarre suficiente para evitar que se caiga el objeto, pero que le permita ser movido con facilidad. Estos movimientos son propios de la mano, son movimientos especializados los cuales implican: 
- aislar

- disociar

- graduar

- calibrar(tener ritmo y fluidez)

Con las características anteriormente mencionadas se permite agarrar el lápiz y después para poder manipular el objeto es necesario según Exner (1989) que el lápiz pueda ser ajustado en las estructuras implicadas, pasarlo de la palma a los dedos y de los dedos a la palma y moverlo entre los mismos dedos:

- desplazar

- trasladar

- rotar

Definitivamente en esta actividad de escribir con la mano, cuyo producto final es la escritura se debe siempre establecer 3 indicadores de competencia y efectividad:

a. La legibilidad de la letra

b. La velocidad de la acción

c. La resistencia de la estructura anatómica

Sin embargo no se pueden olvidar los otros factores que también inciden en el producto final:

- La forma, el tamaño y la alineación de la letra.

- El espacio entre letras y las palabras.

Esto también implica además que el niño debe tener su componente socio-emocional en total equilibrio para que se motive en la actividad de aprender a escribir, que le produzca interés, satisfacción personal, reconocimiento social y por ende un buen nivel de autoestima y auto-concepto.

\section{CONCLUSIONES}

A pesar de los avances tecnológicos, de la magia de los procesadores de palabra y la proliferación de los sistemas de comunicación, la escritura a mano sigue siendo un componente necesario de la vida cotidiana. 
La incapacidad y el resultado final de una escritura ilegible afecta directamente el desempeño académico del estudiante, su autoestima y la aceptación por parte de sus compañeros, maestros y padres.

La pobre coordinación o las inadecuadas habilidades motoras finas no pueden servir como único predictor en la acción de escribir a mano en forma competente, sino que además se deben tener en cuenta los aspectos de procesamiento visual, las habilidades de maduración e integración cognitiva, la memoria visual e integración visual motora.

El otro aspecto a tener en cuenta es que la velocidad de escribir a mano depende tanto de la edad de desarrollo como del entrenamiento que haya tenido en el aula escolar.

Finalmente se recomienda a los Terapeutas Ocupacionales que trabajan con disfunciones de miembro superior, que un aspecto que siempre deben tener en cuenta en sus programas de rehabilitación para medir desempeño ocupacional competente de una persona, es la acción de escribir a mano, analizando cada uno de los factores que intervienen en ésta actividad.

\section{BIBLIOGRAFÍA}

- Burton, A.; Dancisack, M. American journal occupation therapy, vol. 54 No. 1 de 2000.

- Cailliet, R. Síndromes dolorosos de la mano. México: Editorial el manual moderno, 1985.

- Erhardt, R. Development hand dysfunction: Theory, assessment, treatment. Ramsco publishing 1982.

- Flehmig. Desarrollo normal del lactante y sus desuiaciones: diagnostico y tratamientos tempranos. Editorial Panamericana. 1988.

- Gilfoyle, E.; Grady A.; Moore, J. Children Adapt: a theory of sensoriomotorsensory development. 1990. 
- Henderson, Anne; Pehoski, Charlene. Hand function in the child: Foundations for remediation. Edited by Mosby, 1995.

- Kapandji, A. Cuadernos de fisiologia articular. Masson. 1985

- Molina, D.; Costallat, M.OLINA D, COSTALLAT M, La entidad psicomotriz: abordaje de su estudio y su educación. Editado por Losada. 1984.

- Tseng M.; Chow S. Perceptual-motor function of school-age children with slow handwriting speed. The American Journal of Occupational Therapy. Vol. 54 \# 1. 2000.

- Wallen M.; Mackay, S. Test-retest, interracter, realibility and construct validity the handwriting speed test in year 3 and 6 year students.

Physical \& Occupational Therapy in pediatrics, vol. 19 \# 1, 1995.

\section{Desde que su niño nace tiene un programa en el Jardin Infantil}

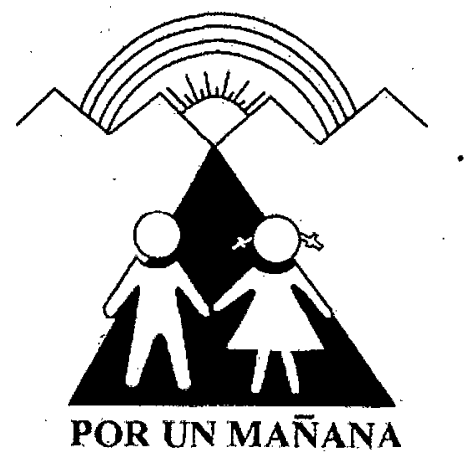

Computudores, Música, Inglés. Apoyo Pedagógico-Terapia Ocupacional Fonoaudiología.

20 AÑOS DE FXPERIFNCIA EN IXSTIMULACION $Y$ PROMOCION DEI, DESARROILO INEANTIL.

Tel: 6714113 - Telefax: 6719986

Transv. 62 No. 174-51 San losé de Bàvaria. 